\title{
Chiral Diamine in Small Molecule Biomimetic Asymmetric Catalysis
}

Fanyun Zeng ${ }^{1} \dagger$, Chen $\mathrm{Chen}^{1} \uparrow$, Liu Liu ${ }^{1} \dagger$, Yifan $\mathrm{Li}^{1} \uparrow, \mathrm{Bo} \mathrm{Li}^{2}$, Guotao Lin ${ }^{1}$, Shihao Huang ${ }^{1}$, Mingyu Zhang ${ }^{1}$, Yuting Yan $^{1}$, Yuan Zhang ${ }^{1}$, Yanfeng Dang ${ }^{2} *$, Jianhui Huang ${ }^{1} *$

\section{Affiliations:}

${ }^{1}$ Tianjin Key Laboratory for Modern Drug Delivery \& High-Efficiency; School of Pharmaceutical Science and Technology, Tianjin University; Tianjin, 300072, China.

${ }^{2}$ Tianjin Key Laboratory of Molecular Optoelectronic Science, Department of Chemistry, School of Science, Tianjin University; Tianjin, 300072, China.

*Corresponding author. Email: yanfengdang@tju.edu.cn; jhuang@tju.edu.cn.

$\dagger$ These authors contributed equally to this work.

th memory of Professors Jonathan Williams and Peter Beak.

Abstract: Enzymatic reaction, as an environmentally friendly approach, has made great progress producing commodity chemicals comparing to the conventional metallo/organo catalysis. However, the reaction compatibility is not satisfactory. The development of biomimetic catalysis balancing both strategies for the green and broad application in synthesis is desirable. Here, we report the design and synthesis of a chiral diamine catalyst fulfilling this requirement. Asymmetric addition reactions using this ligand in water were demonstrated and the corresponding products were produced in excellent yields and enantiomeric ratios. This pluripotent ligand has also shown good reactivity/enantioselectivity on a number of representative reactions in both green and organic solvents. We anticipate that the ligand would allow further development of other catalysts for important yet challenging green stereoselective transformations.

25 One-Sentence Summary: A pluripotent chiral diamine catalyst is discovered for the efficient asymmetric catalysis in water.

From feedstock to commodity chemicals and further to biological and pharmaceutical active ingredients, asymmetric synthesis has paved the way to access molecules covering spacious chemical space and structure diversity/complexity (1). Enzymatic reactions comparing to organic reactions are generally more efficient with regard to the features such as catalytic, high atom economical, eco-friendly, highly reactive and high stereoselective but with limited reaction compatibility (2). (Fig. 1A) Whereas in organic synthesis, metallo- or organo- catalysts have broad applications in various reaction pathways with relative moderate efficiency, but most of the catalyses were carried out in organic solvents (3). Biomimetic reactions, including reactions using tailored enzymes (4) and small-molecule biomimetic catalysis (5) as a combined conceptual breakthrough bridging both bio- and small molecule catalyses covers broad reaction compatibility and utilizing environmentally benign agents $(6)$. We would like to develop small-molecule catalysts and mimic addition reactions initially catalysed by enzymes (7-9). 

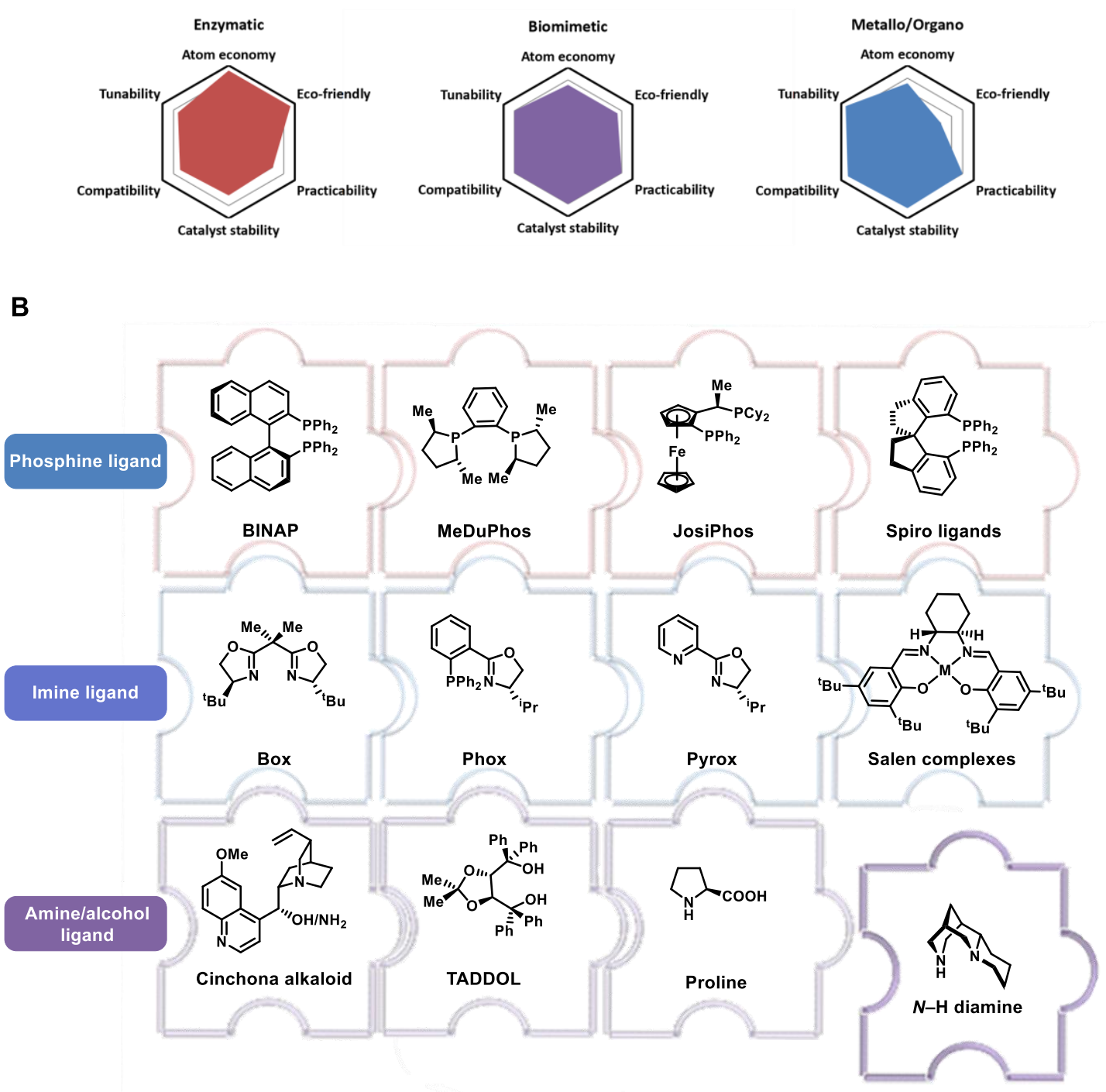

Fig. 1. Typical catalyses and privileged organic catalysts. (A) Biomimetic catalysis, the bridge of enzymatic and metallo/organo catalyses. (B) Privileged ligands and new candidates.

In small molecule catalysis, commonly recognized privileged chiral catalysts $(10,11)$ as summarized by Jacobsen and Zhou have shown in Fig. 1B. BINAP, MeDuPhos, JosiPhos, Spiro ligands are phosphine-based ligands; Box, Phox, Pyrox, Salen complexes are typical imine containing ligands; Cinchona alkaloid, TADDOL, Proline are a class of amine/alcohol ligand, which has been well studied in the past for asymmetric catalysis mainly in organic solvents. The utilization of environmentally benign catalyst/ligand allowing stereoselective transformations in green fashion with unnecessary derivatization is the ultimate desire.

Naturally occurring product sparteine with both enantiomers, (+)-sparteine and

15 (-)-sparteine with a tetracyclic skeleton, was one of the most well-known alkaloids used in asymmetric synthesis since 1970s. Leading by Noyori $(12,13)$ and Trost (14), reactions utilizing commercially available chiral diamines have been examined. Later contributions using sparteine with Li (15-27), Mg (28), Ni (29), Cu (30-32), Zn (33) 
and $\mathrm{Pd}(34-38)$ reagents have also provided broad applications in asymmetric synthesis. However, due to the less available analogs, other than organolithium reactions, the enantioselectivities using sparteine under mechanistically unrelated reactions were less successful comparing to the well-recognized privileged chiral catalysts.

5 Here, we report a computer-aided ligand design and the access of a low toxic $\mathrm{N}-\mathrm{H}$ diamine analogue based on sparteine for enantioselective biomimetic catalysis. The selected asymmetric reactions have shown that the chiral ligand has high reactivity and stereoselectivity with broad applications on a number of reactions in green and organic solvents. The allowance of a family of ligands with functionality at various positions was also demonstrated on asymmetric addition reaction with toxicity evaluations.

Inspired by the excellent potential features of sparteine, a tertiary diamine with broad application in various reactions, we started our design in minimalism principle relied on the computational studies of sparteine. Quantum mechanical computations were applied to engineer the sparteine scaffold to achieve better performance. Intending the scaffold optimization to be more general, we sought to tailor the ligand environment for reaction events in the first coordination sphere, which we recognize as a major challenge, by focusing on a representative inner-sphere process by Pyrox-Pd catalysis (39), i.e. the asymmetric addition of $N$-Ts imine with $\mathrm{PhB}(\mathrm{OH})_{2}$ (Fig. 2A). Computational studies led us to establish a plausible pathway consisting of transmetalation (TS1), migratory insertion (TS2), and protonation (TS3) (40). The resulting free energy profile drew our attention to the rate-limiting and enantio-controlling migratory insertion step. Two clues for ligand design were implicated by an analysis of TS2. First, this sterically congested transition state, displaying multiple ligand-substrate repulsions (Fig. 2B), could be stabilized by decreasing peripheral hindrances (e.g. rings 1 and 2), providing a means of accelerating the catalytic process. Second, the computed stereoisomeric transition states suggest that the barrier difference of TS2a vs TS2b is significantly larger than TS2a' vs TS2b' (Fig. $2 \mathrm{C})$. We reasoned that an arrangement where the more anisotropic $N$-Ts imine is placed on the sterically biased right-hand side of Sparteine (i.e. as in TS2a/TS2b; see Fig. 2D for a steric contour) would be desirable for maximizing stereodifferentiation. 


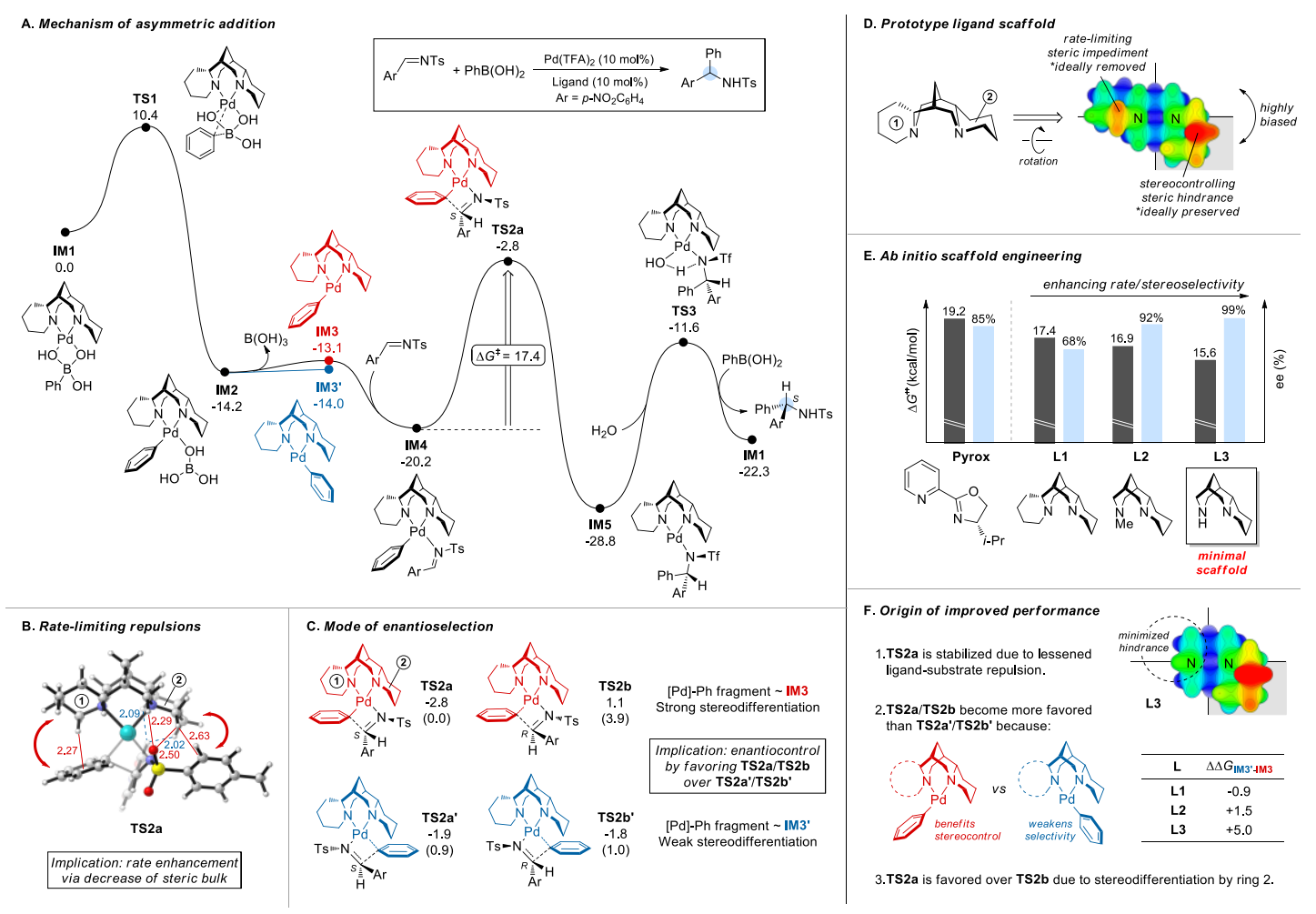

Fig. 2. Computational ligand design. (A) Mechanism of $\mathrm{Pd} /$ sparteine-catalysed asymmetric addition. (B) Rate-limiting steric repulsions. (C) Mode of enantioselection. (D) Prototype ligand scaffold. (E) Ab initio scaffold engineering. (F) Origin of improved performance. Free energies are shown in $\mathrm{kcal} / \mathrm{mol}$, relative values in parentheses. More computational details are provided in the Supporting Information.

We tested our hypotheses by computationally assessing the influence of removing ring 1 from sparteine (Fig.s 2D and 2E). To our delight, it was demonstrated by L1-L3 that a significant improvement can be realized by our backbone engineering. The computationally derived ligand $\mathbf{L} \mathbf{3}$, which preserves the rigid bispidine core but can better accommodate and exert stereocontrol over the reactive fragments (Fig. 2F), gives a low barrier of $15.6 \mathrm{kcal} / \mathrm{mol}$ and an excellent ee of $99 \%$, both superior to the prototype $\mathbf{L 1}$ as well as the Pyrox privileged ligand. The effectiveness of $\mathbf{L 3}$ implies a strong

15 stereodiscrimination over inner-sphere transformations from a minimal chiral scaffold, illustrating the promising potential of sparteine-family ligands for a broader scope of asymmetric catalysis.

Based on the computational studies above as well as rationale from Kozlowski and Gaunt on $N-\mathrm{H}$ containing amine ligands $(41,42), N-$ Me diamine $\mathbf{L} 2$ also known as O'Brien diamine and $N-\mathrm{H}$ diamine $\mathbf{L} \mathbf{3}$ have been prepared with modified procedures based on the literature precedence. (see SI, Scheme S1) (43-46) Starting from commercially available alkaloid (-)-cytisine, (47) both diamines were successfully prepared in $>30$ gram scale and are ready for the further assessments on asymmetric reaction under sustainable conditions. The structure of bench-stable $\mathbf{L 3} \cdot \mathrm{HCl}$ salt was ambiguously determined by X-ray single crystal diffraction (CCDC No.2116108). Reactions of cyclic and acyclic imines with 4-methoxylphenyl boronic acid in trifluoroethanol have been studied first. Even though in the literature, these diamine ligands were not considered as good ligands for reactions out of organolithium field 
$(48,49)$. Both of diamines $\mathbf{L} 2$ and $\mathbf{L} 3$ have provided excellent reactivity and enantioseletivity for all of the four reactions comparing to the moderate enantiomeric ratios generated from (+)-sparteine as shown in Fig. 2A (50). Interestingly, for the addition to sterically hindered $N$-sulfonyl ketimines, ligand $\mathbf{L 3}$ provided the corresponding sulfonamide $\mathbf{2}$ in $90 \%$ yield with $96: 4$ er while $N-$ Me diamine ligand $\mathbf{L} 2$ provided the product in 67\% yield with reduced er (69:31), while the use of (+)-sparteine gave diminished enantioselectivity (50:50 er).

The green synthesis of active pharmaceutical ingredients is important especially on industrial scale production. The efforts on the addition reactions under green processes were also carried out. In water, the same reaction using known privileged catalysts such as BINAP L4, Phox L5, (51-53) Pyrox L6 (54) as well as proline $\mathbf{L} 7$ together with O'Brien diamine $\mathbf{L} 2, N-\mathrm{H}$ enzyme mimic $\mathbf{L} 3$ were carried out. Under similar conditions, the desired product 1 was obtained with yields and er.s have been listed in Fig. 3B. BINAP L4 provided good stereoselctivity of 95:5 er but moderate yield of $40 \%$ 15 due to the oxidation of phosphine during the reaction; Phox gave the corresponding product 1 with good yield and er; Pyrox and Proline were not efficient chiral catalysts for this reaction in water even with good reaction yields. Whereas diamine $\mathbf{L} \mathbf{2}$ provided the sulfonamide $\mathbf{1}$ with good yield and good er, $N-\mathrm{H}$ diamine $\mathbf{L} \mathbf{3}$ gave the product with nearly quantitative yield with excellent er. (Fig. 2B)

20 With the conditions in hands, we have examined the reactions employing $N-\mathrm{Me}$ diamine $\mathbf{L} 2$ or $N-\mathrm{H}$ diamine $\mathbf{L 3}$. Reactions of arylboronic acids with 5-membered cyclic sulfonyl imides were also very successful in water. A group of representative sulfonamides 5-13 have been prepared with both $N-\mathrm{H}$ and $N$-Me ligands under similar conditions. Very encouragingly, among all the examples demonstrated, $N-\mathrm{H}$ diamine

$25 \mathbf{L} \mathbf{3}$ and O'Brien diamine $\mathbf{L} \mathbf{2}$ had shown excellent reactivity and stereoselectivity, and all of the desired products were obtained in excellent yields and enantiomeric ratios. (Fig. 3C) 


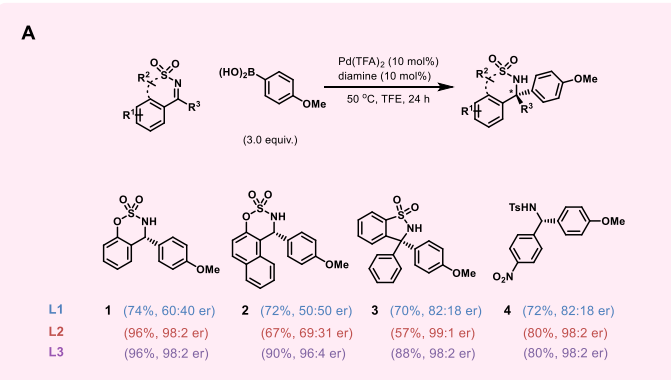

B

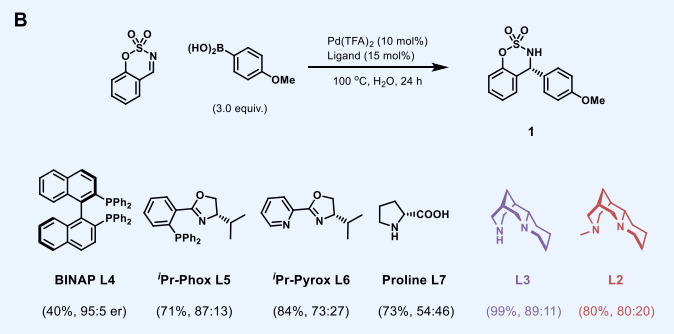

C

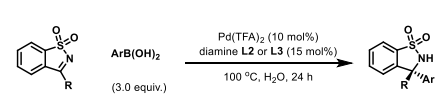

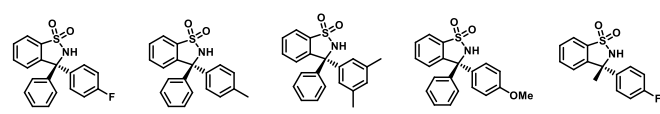

$\begin{array}{rrrrr}\mathbf{5}(79 \% \%, 2: 98 \mathrm{er}) & \mathbf{6}(83 \%, 97: 3 \mathrm{er}) & \mathbf{7}(80 \%, 3: 97 \mathrm{er}) & \mathbf{3}(88 \%, 2: 98 \mathrm{er}) & \mathbf{8}(88 \%, 97: 3 \mathrm{er}) \\ (64 \%, 3: 97 \mathrm{er}) & (85 \%, 97: 3 \mathrm{er}) & (80 \%, 8: 92 \mathrm{er}) & (80 \%, 16: 84 \mathrm{er}) & (85 \%, 97: 3 \mathrm{er})\end{array}$

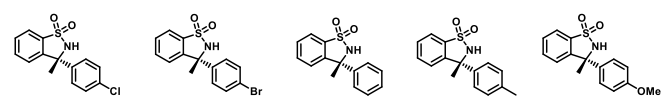

$\begin{array}{rrrrr}\mathbf{9}(89 \%, 98: 2 \mathrm{er}) & \mathbf{1 0}(75 \%, 98: 2 \mathrm{er}) & \mathbf{1 1}(95 \%, 97: 3 \mathrm{er}) & \mathbf{1 2}(92 \%, 97: 3 \mathrm{er}) & \mathbf{1 3}(93 \%, 97: 3 \mathrm{er}) \\ (91 \%, 97: 3 \mathrm{er}) & (73 \%, 91: 9 \mathrm{er}) & (89 \%, 95: 5 \mathrm{er}) & (90 \%, 96: 4 \mathrm{er}) & (90 \%, 95: 5 \mathrm{er})\end{array}$

$$
\text { (3.0 equiv.) }
$$

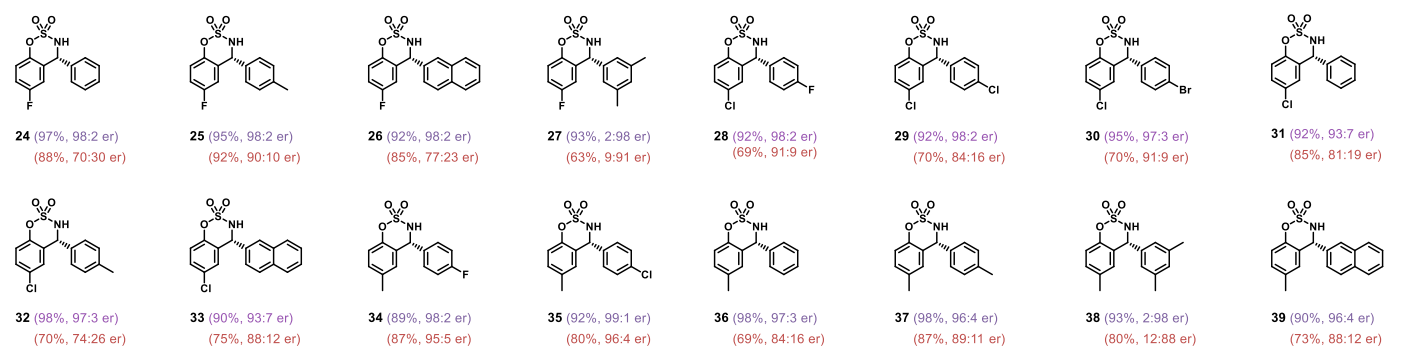

(6)

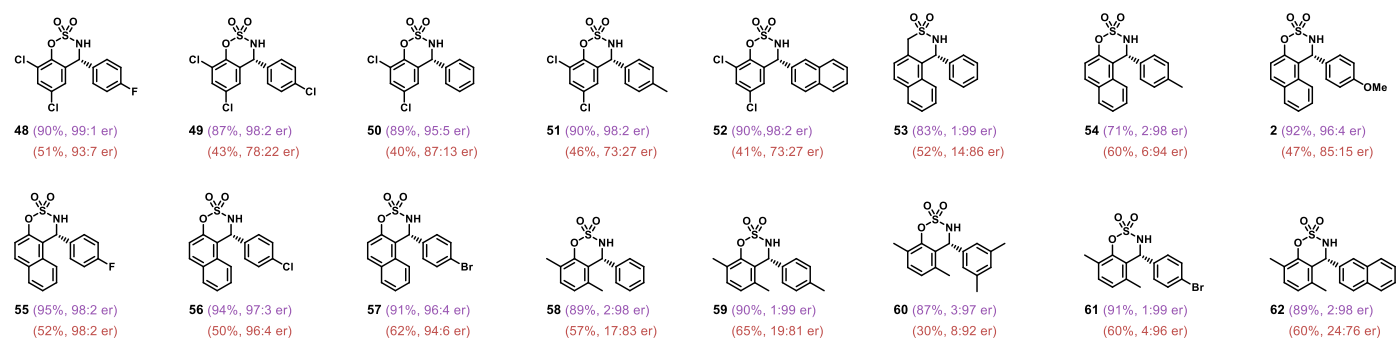

Fig. 3. Green synthesis of chiral sulfonamides. (A) Ligand screening in organic solvent. (B) Ligand screening in water. (C) Reaction scope with chiral diamine ligands $\mathbf{L} \mathbf{2}$ or $\mathbf{L} \mathbf{3}$ in water.

5 Reactions of arylboronic acids with 6-membered cyclic $N$-sulfonyl ketimides were also fruitful, a large number of corresponding sulfonamides have been prepared with either diamines $\mathbf{L} \mathbf{2}$ or $\mathbf{L 3}$. (Fig. 3C) We were pleased to find in all the cases, $N-\mathrm{H}$ diamine $\mathbf{L 3}$ gave higher yields with higher enantiomeric ratios comparing to $\mathrm{N}-\mathrm{Me}$ diamine $\mathbf{L} 2$. Particularly for reactions with sterically hindered nucleophiles such as naphthyl boronic acid, the er values for $N$-Me were generally much lower than the other reactions. The formation of sulfonamide 19 was very positive using $N-\mathrm{H}$ diamine instead of $N-\mathrm{Me}$ ligand while the er values of reactions with two ligands were 11:89 
and 42:58 respectively. A large number of representative examples have been demonstrated and the corresponding chiral sulfonamides were obtained in good yields and enantioselectivity.

Not only the 1,2-addition reaction, the reactions of arylboronic acid under Pd catalysed conditions were also very successful for the Michael addition on enones (Fig. 4A). The corresponding products were also obtained in good yields and ers. Comparing to the known ligand ${ }^{i}$ Pr-Pyrox (70:30 er) reported by Stoltz (40), our ligand provided the product with higher er even with the simple skeleton (13 atoms) before the introduction of sterically enhancing group.

10 To evaluate the ligand further, a number of representative reactions including organocatalysis, $\mathrm{Cu}$-catalysed Henry reaction and oxidative coupling reaction; Pd-catalysed hydroarylation and kinetic oxidation reactions were examined. Under un-optimized conditions, the reactions provided the corresponding products with good yields and good enantiomeric ratios. As shown in Fig. 4B, organocatalysis under neat conditions, the desired chiral alcohol 71 was obtained in 79\% yield with 83:17 dr and 98:2 er. Binol derivative $\mathbf{7 3}$ was also successfully prepared in 71\% yield and 96:4 er (55). More interestingly, reaction of iodobenzene with norbornene, in the presence of Pd catalyst and diamine ligand $\mathbf{L 3}$, the desired phenyl substituted norbonane $\mathbf{7 7}$ was prepared in 95:5 er whereas in the literature, the best enantioselectivity was achieved under Zhou's conditions using Quinox ligand at 87:13 er (56). (Fig. 4B, eq. 4) A standard Henry rection using aldehyde 69 with nitromethane provided the corresponding chiral alcohol $\mathbf{7 4}$ in 77\% yield with 90:10 er in ethanol (57). In water, under conditions similar to Stoltz and Sigman reported 20 years ago $(36,37)$, the kinetic oxidation reaction was also successful and alcohol $\mathbf{7 8 - R}$ was obtained in $43 \%$ yield with 76:24 er.

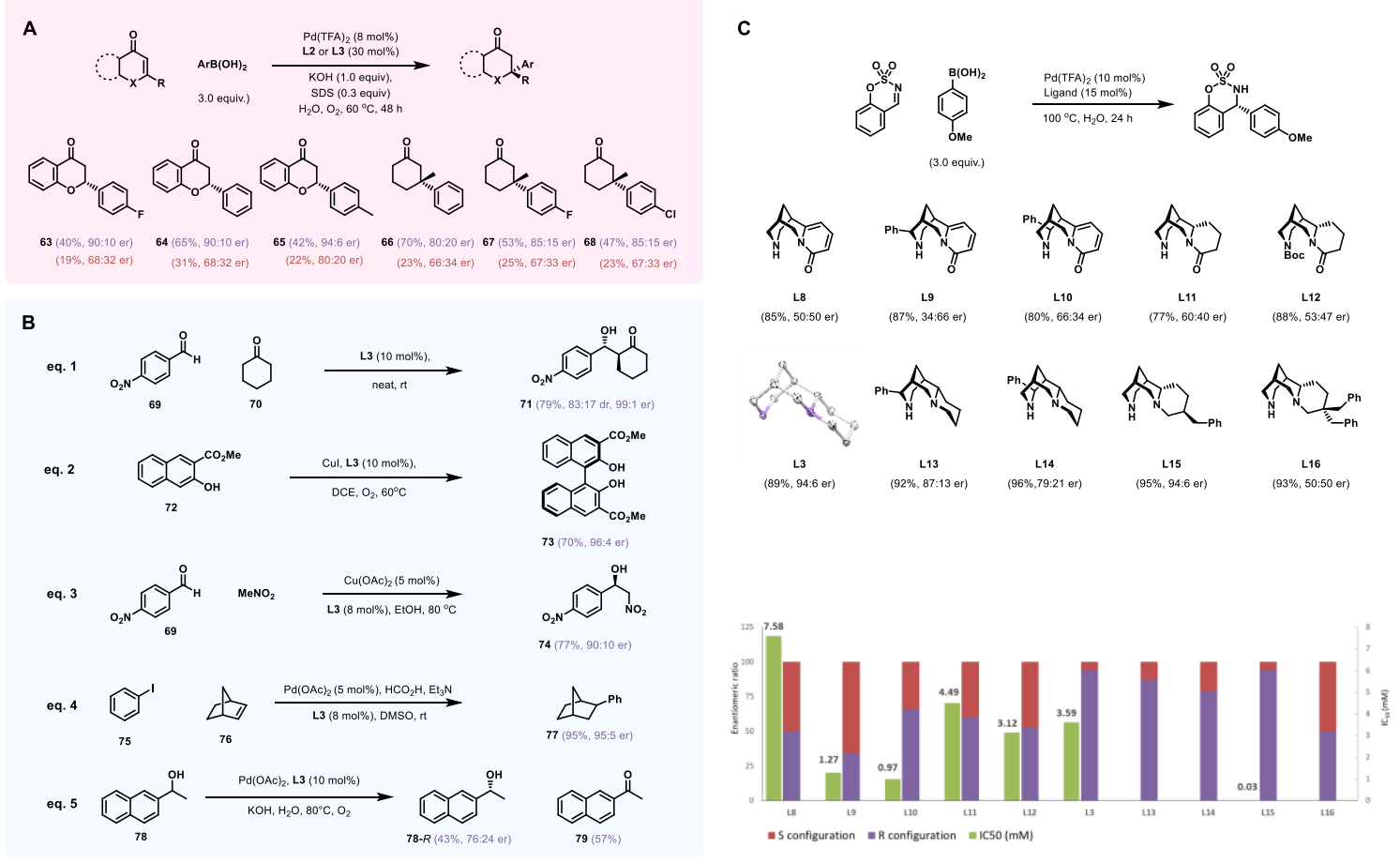

Fig. 4. Ligand evaluation on various mechanistically irrelevant reactions. (A) Asymmetric Michael addition in water. (B) Miscellaneous reactions using diamine 
ligand L3. (C) Diamine ligand modifications, evaluation and toxicity studies against HEK293T cells.

Similar chiral diamine ligands within the family have been prepared and evaluated against a model reaction in water. Under standard conditions, ligands L3 and L15 have shown similar high asymmetric induction, while the introduction of groups on (-)-cytisine derivatives provided sufonamide 1 with good yields but low er.s. Ligands such as L8, L10 and L12 have shown reduced enantioselectivity; L9 and L10 have shown opposite asymmetric induction due to the substituent on the other site of (-)-cytisine.

10 With regard to green catalysts, the toxicity studies were also carried out for selected chiral $\mathrm{N}-\mathrm{H}$ diamines in order to reveal the green profiles for the future ligand development. Using HEK293T cell, these ligands have been evaluated. Diamine L8, as a smoking cessation agent, showed the lowest toxicity among theses diamines (58) (Fig 4C, green bar). Ligands L3, L11, L12 showed similar low toxicity to (-)-cytisine even

15 though $\mathbf{L 1 1}$ and $\mathbf{L 1 2}$ provided the reactions with low er.s. Unfortunately, ligand $\mathbf{L 1 5}$ with good reactivity and enantioselectivity has shown over 200 times higher toxicity comparing to (-)-cytisine. (see SI table S1). It is delightful that our pluoripotent chiral diamine ligand $\mathbf{L} \mathbf{3}$ showed low cytotoxicity to become a possible ligand in green chemistry.

20 We have developed a new class of chiral $\mathrm{N}-\mathrm{H}$ diamine ligands for biomimetic catalysis. The results highlight the substantial scope of the chiral catalysts in green asymmetric synthesis especially in the efforts producing pharmaceutically important agents. The new family members with new reactivity and selectivity would be worthwhile studying in the future.

\section{References and Notes}

1. G.-Q. Lin, Q.-D. You, J.-F. Cheng, Eds., Chiral Drugs (Wiley-VCH, Weinheim, 2011).

2. K. Chen, F. H. Arnold, Engineering new catalytic activities in enzymes. Nat. Catal. 3, 203-213 (2020).

3. G. L. D. Krupadanam, Ed. Fundamentals of Asymmetric Synthesis (CRC press, London, 2014).

4. S. Wu, R. Snajdrova, J. C. Moore, K. Baldenius, U. T. Bornscheuer, Angew. Chem. Int. Ed. 60, 88-119 (2021).

5. P. I. Dalko, Ed. Comprehensive Enantioselective Organocatalysis: Catalysts, Reactions, and Applications (Wiley-VCH, Weinheim, 2013).

6. G. I. Likhtenshtein, New Trends in Enzyme Catalysis and Biomimetic Chemical Reactions (Springer, Boston, 2007).

7. Y. Zhang, R. Li, Y. He, Z. Guan, Bio-catalytic bis-Michael reaction for generating cyclohexanones with a quaternary carbon center using glucoamylase, Catal. Lett. 147, 633-639 (2017).

8. I. Janicki, P. Łyżwa, P. Kiełbasiński, The first enzyme-promoted addition of nitromethane to imines (aza-Henry reaction), Bioorg. Chem. 94, 10377 (2020). 
9. J. H. Schrittwieser, S. Velikogne, W. Kroutil, Biocatalytic imine reduction and reductive amination of ketones. Adv. Synth. Catal. 357, 1655-1685 (2015).

10. T. P. Yoon, E. N. Jacobsen, Privileged chiral catalysts. Science 199, 1691-1693 (2003).

11. Q.-L. Zhou, Ed. Privileged Chiral Ligands and Catalysts (Wiley-VCH, Weinheim, 2011).

12. H. Nozaki, T. Aratani, T. Toraya, R. Noyori, Asymmetric ring opening of gem-dibromocyclopropanes leading to allenic hydrocarbons. Tetrahedron Lett. 9, 2087-2090 (1968).

10 13. H. Nozaki, T. Aratani, T. Toraya, R. Noyori, Asymmetric syntheses by means of (-)-sparteine modified organometallic reagents. Tetrahedron 27, 905-913 (1971).

14. B. Trost, T. Dietsche, New synthetic reactions. Allylic alkylations asymmetric induction in allylic alkylations. J. Am. Chem. Soc. 95, 8200-8201 (1973).

15. D. Hoppe, F. Hintze, P. Tebben, Chiral lithium-1-oxyalkanides by asymmetric deprotonation; enantioselective synthesis of 2-hydroxyalkanoic acids and secondary alkanols. Angew. Chem. Int. Ed. 29, 1422-1424 (1990).

16. S. T. Kerrick, P. Beak, Asymmetric deprotonations: Enantioselective syntheses of 2-substituted (tert-butoxycarbonyl)pyrrolidines. J. Am. Chem. Soc. 113, 9708-9710 (1991).

20 17. M. Lautens, C. Gajda, P. Chiu, Studies in the asymmetric ring opening of an oxabicyclic compound. Catalytic asymmetric induction using (-)-sparteine. $J$. Chem. Soc. Chem. Commun. 1193-1194 (1993).

18. S. E. Denmark, N. Nakajima, O. J.-C. Nicaise, Asymmetric addition of organolithium reagents to imines. J. Am. Chem. Soc. 116, 8797-8798 (1994).

19. S. Klein, I. Marek, J.-F. Poisson, J.-F. Normant, Asymmetric carbolithiation of cinnamyl derivatives in the presence of (-)-sparteine. J. Am. Chem. Soc. 117, 88538854 (1995).

20. A. R. Muci, K. R. Campos, D. A. Evans, Enantioselective deprotonation as a vehicle for the asymmetric synthesis of $\mathrm{Cz}$-symmetric $\mathrm{P}$-chiral diphosphines. J. Am. Chem. Soc. 117, 9075-9076 (1995).

21. M. Tsukazaki, M. Tinkl, A. Toglans, B. J. Chapell, N. J. Taylor, V. Snieckus, Direct and highly enantioselective synthesis of ferrocenes with planar chirality by (-)-sparteine-mediated lithiation. J. Am. Chem. Soc. 118, 685-686 (1996).

22. K. R. Campos, A. Klapars, J. H. Waldman, P. G. Dormer, C.-y. Chen, Enantioselective, palladium-catalyzed $\alpha$-arylation of $N$-Boc-pyrrolidine $J$. Am. Chem. Soc. 128, 3538-3539 (2006).

23. C. Genet, S. J. Canipa, P. O'Brien, S. Taylor, Catalytic asymmetric synthesis of ferrocenes and P-stereogenic bisphosphines. J. Am. Chem. Soc. 128, 9336-9337 (2006).

24. N. S. Sheikh, D. Leonori, G. Barker, J. D. Firth, K. R. Campos, P. O’Brien, I. Coldham, An experimental and in situ IR spectroscopic study of the lithiationsubstitution of $N$-Boc-2-phenylpyrrolidine and -piperidine: controlling the formation of quaternary stereocenters. J. Am. Chem. Soc. 134, 5300-5308 (2012). 
25. M. Burns, S. Essafi, J. Bame, S. P. Bull, M. P. Webster, S. Balieu, J. W. Dale, C. P. Butts, J. N. Harvey, V. K. Aggarwal, Assembly-line synthesis of organic molecules with tailored shapes. Nature 513, 183-188 (2014).

26. W. Lin, K.-F. Zhang, O. Baudoin, O. Regiodivergent enantioselective C-H functionalization of Boc-1,3-oxazinanes for the synthesis of $\beta 2$ - and $\beta 3$-amino acids. Nat. Catal. 2, 882-888 (2019).

27. S. Monticelli, W. Holzer, T. Langer, A. Roller, B. Olofsson, V. Pace, Sustainable asymmetric organolithium chemistry: enantio- and chemoselective acylations through recycling of solvent, sparteine, and Weinreb "Amine". ChemSusChem 12, 1147-1154 (2019).

28. R. Shintani, G. C. Fu, Highly enantioselective desymmetrization of anhydrides by carbon nucleophiles: reactions of Grignard reagents in the presence of (-)-sparteine. Angew. Chem. Int. Ed. 41, 1057-1059 (2002).

29. H. Brunner, H. B. Kagan, G. Kreutzer, Asymmetric catalysis. Part 153: Metal-catalysed enantioselective $\alpha$-ketol rearrangement. Tetrahedron: Asymmetry 14, 2177-2187 (2003).

30. M. Smrčina, J. Poláková, S. Vyskočil, P. Kočovský, Synthesis of enantiomerically pure binaphthyl derivatives. Mechanism of the enantioselective, oxidative coupling of naphthols and designing a catalytic cycle. J. Org. Chem. 58, 4534-4538 (1993).

20 31. D. Nakamura, K. Kakiuchi, K. Koga, R. Shira, Design and synthesis of novel C2-symmetric chiral piperazines and an application to asymmetric acylation of б-symmetric 1,2-diols. Org. Lett. 8, 6139-6142 (2006).

32. G. Hu, D. Holmes, B. F. Gendhar, W. D. Wulff, Optically active ( $a R)$ - and $(a S)$-linear and vaulted biaryl ligands: deracemization versus oxidative dimerization. J. Am. Chem. Soc. 131, 14355-14364 (2009).

33. H. Mimoun, J. Y. de Saint Laumer, L. Giannini, R. Scopelliti, C. Floriani, Enantioselective reduction of ketones by polymethylhydrosiloxane in the presence of chiral Zinc catalysts. J. Am. Chem. Soc. 121, 6158-6166 (1999).

34. A. Togni, Asymmetric allylic alkylation catalyzed by palladium-sparteine complexes. Tetrahedron: Asymmetry 2, 683-690 (1991).

35. J. Kang, W. O. Cho, H. G. Cho, (-)- $\alpha$-Isosparteine as a chiral ligand in asymmetric allylic alkylation. Tetrahedron: Asymmetry 5, 1347-1352 (1994).

36. D. R. Jensen, J. S. Pugsley, M. S. Sigman, Palladium-catalyzed enantioselective oxidations of alcohols using molecular oxygen. J. Am. Chem. Soc. 123, 7475-7476 35 (2001).

37. E. M. Ferreira, B. M. Stoltz, The Palladium-catalyzed oxidative kinetic resolution of secondary alcohols with molecular oxygen. J. Am. Chem. Soc. 123, 7725-7726 (2001).

38. K.-T. Yip, M. Yang, K.-L. Law, N.-Y. Zhu, D. Yang, Pd(II)-catalyzed enantioselective oxidative tandem cyclization reactions. synthesis of indolines through C-N and C-C bond formation. J. Am. Chem. Soc. 128, 3130-3131 (2006).

39. H. Dai, X. Lu, Palladium (II)/2,2'-bipyridine-catalysed addition of arylboronic acids to $N$-tosyl arylaldimines. Tetrahedron Lett. 50, 3478-3481 (2009). 
40. J. C. Holder, L. Zou, A. N. Marziale, P. Liu, Y. Lan, M. Gatti, K. Kikushima, K. N. Houk, B. M. Stoltz, Mechanism and enantioselectivity in Palladium-catalyzed conjugate addition of arylboronic acids to $\beta$-substituted cyclic enones insights from computation and experiment. J. Am. Chem. Soc. 135, 14996-15007 (2013).

41. P.-W. Phuan, J. C. Ianni, M. C. Kozlowski, Is the A-ring of sparteine essential for high enantioselectivity in the asymmetric lithiation-substitution of N-Boc-pyrrolidine? J. Am. Chem. Soc. 126, 15473-15479 (2004).

42. A. McNally, B. Haffemayer, B. Collins, M. J. Gaunt, Palladium-catalysed C-H activation of aliphatic amines to give strained nitrogen heterocycles. Nature 510, 129-133 (2014).

43. M. Freund, P. Horkheimer, Zur kenntniss des Cytisins. Ber. Dtsch. Chem. Ges. 39, 814-825 (1906).

44. P. Scheiber, P. Nemes, Synthesis and stereochemistry of some new diazatricyclic compounds. Liebigs Ann. Chem. 10, 1033-1036 (1994).

15 45. M. J. Dearden, C. R. Firkin, J.-P. R. Hermet, P. O’Brien, A readily-accessible (+)-sparteine surrogate. J. Am. Chem. Soc. 124, 11870-11871 (2002).

46. D. Ebner, thesis, California Institute of Technology (2009).

47. J. Rouden, M.-C. Lasne, J. Blanchet, J. Baudoux. (-)-Cytisine and derivatives: synthesis, reactivity, and applications. Chem. Rev. 114, 712-778 (2014).

48. J. P. Michael, Indolizidine and quinolizidine alkaloids. Nat. Prod. Rep. 24, 191222 (2007)

49. O. O. Grygorenko, D. S. Radchenko, D. M. Colochnyuk, A. A. Tolmachev, I. V. Komarov, Bicyclic conformationally restricted diamines. Chem. Rev. 111, 55065568 (2011).

50. C. Jiang, Y. Lu, T. Hayashi, High performance of a palladium phosphinooxazoline catalyst in the asymmetric arylation of cyclic $N$-sulfonyl ketimines. Angew. Chem. Int. Ed. 53, 9936-9939 (2014).

51. G. J. Dawson, C. G. Frost, J. M. J. Williams, S. J. Coote, Asymmetric palladium catalysed allylic substitution using phosphorus containing oxazoline ligands. Tetrahedron Lett. 34, 3149-3150 (1993).

52. P. von Matt, A. Pfaltz, Chiral phosphinoaryldihydrooxazolesas ligands in asymmetric catalysis: Pd-catalyzed allylic substitution. Angew. Chem. Int. Ed. 36, 566-568 (1993).

53. J. Sprinz, G. Helmchen, Phosphinoaryl- and phosphinoalkyloxazolines as new chiral ligands for enantioselective catalysis: very high enantioselectivity in palladium catalysed allylic substitutions. Tetrahedron Lett. 34, 1769-1772 (1993).

54. H. Brunner, U. Obermann, P. Wimmer, Asymmetrische katalysen: XXXII. Enantioselektive phenylierung von cis-cyclohexan-1,2-diol und meso-butan-2,3-diol. J. Organomet. Chem. 316, C1-C3 (1986).

55. N. Mase, Y. Nakai, N. Ohara, H. Yoda, K. Takabe, F. Tanaka, C. F. Barbas III, Organocatalytic direct asymmetric aldol reactions in water. J. Am. Chem. Soc. 128, 734-735 (2006). 
56. X.-Y. Wu, H.-D. Xu, Q.-L. Zhou, A. S. C. Chan, Enantioselective Heck-type hydroarylation of norbornene with phenyl iodide catalyzed by palladium/quinolinyl oxazolines. Tetrahedron: Asymmetry 11, 1255-1257 (2000).

57. H. Maheswaran, K. L. Prasanth, G. G. Krishna, K. Ravikumar, B. Sridhar, M. L. Kantam, Enantioselective nitroaldol (Henry) reaction using copper(II) complexes of (-)-sparteine. Chem. Commun. 4066-4068 (2006).

58. H. R. Campello, S. G. Del Villar, A. Honraedt, T. Minguez, A. S. F. Oliveira, K. E. Ranaghan, D. K. Shoemark, I. Bermudez, C. Gotti, R. B. Sessions, A. J. Mulholland, S. Wonnacott, T. Gallagher, Unlocking nicotinic selectivity via direct $\mathrm{C}-\mathrm{H}$ functionalization of (-)-cytisine. Chem 4, 1710-1715 (2018).

59. K. Hajime, O. Shigeru, M. Isamu, Absolute stereochemistry of (-)-carnoensine and (-)-camoensidine in Maackia species. Can. J. Chem. 72, 214-217 (1994).

60. T. Jiang, Z. Wang, M. H. Xu, Rhodium-catalyzed asymmetric arylation of cyclic $N$-sulfonyl aryl alkyl ketimines: efficient access to highly enantioenriched $\alpha$-tertiary amines. Org. Lett. 17, 528-531 (2015).

61. Q. He, C. M. So, Z. Bian, T. Hayashi, J. Wang, Rhodium/chiral diene-catalyzed asymmetric 1,4-addition of arylboronic acids to chromones: a highly enantioselective pathway for accessing chiral flavanones. Chem. Asian J. 10, 540543 (2015).

20 62. J. K. Matsui, G. A. Molander, Direct $\alpha$-arylation/heteroarylation of 2-trifluoroboratochromanones via photoredox/nickel dual catalysis. Org. Lett. 19, 436-439 (2017).

63. K. Kikushima, J. C. Holder, M. Gatti, B. M. Stoltz, Palladium-catalyzed asymmetric conjugate addition of arylboronic acids to five-, six-, and seven-membered $\beta$-substituted cyclic enones: enantioselective construction of all-carbon quaternary stereocenters. J. Am. Chem. Soc. 133, 6902-6905 (2011).

64. M. J. Frisch, G. W. Trucks, H. B. Schlegel, G. E. Scuseria, M. A. Robb, J. R. Cheeseman, G. Scalmani, V. Barone, G. A. Petersson, H. Nakatsuji, X. Li, M. Caricato, A. Marenich, J. Bloino, B. G. Janesko, R. Gomperts, B. Mennucci, H. P. Hratchian, J. V. Ortiz, A. F. Izmaylov, J. L. Sonnenberg, D. Williams-Young, F. Ding, F. Lipparini, F. Egidi, J. Goings, B. Peng, A. Petrone, T. Henderson, D. Ranasinghe, V. G. Zakrzewski, J. Gao, N. Rega, G. Zheng, W. Liang, M. Hada, M. Ehara, K. Toyota, R. Fukuda, J. Hasegawa, M. Ishida, T. Nakajima, Y. Honda, O. Kitao, H. Nakai, T. Vreven, K. Throssell, J. A. Montgomery, Jr., J. E. Peralta, F. Ogliaro, M. Bearpark, J. J. Heyd, E. Brothers, K. N. Kudin, V. N. Staroverov, T. Keith, R. Kobayashi, J. Normand, K. Raghavachari, A. Rendell, J. C. Burant, S. S. Iyengar, J. Tomasi, M. Cossi, J. M. Millam, M. Klene, C. Adamo, R. Cammi, J. W. Ochterski, R. L. Martin, K. Morokuma, O. Farkas, J. B. Foresman, and D. J. Fox, Gaussian, Inc., Wallingford CT, 2016.

65. Y. Zhao, D. G. Truhlar, Density functionals with broad applicability in chemistry. 
Acc. Chem. Res. 41, 157-167 (2008).

66. Y. Zhao, D. G. Truhlar, The M06 suite of density functionals for main group thermochemistry, thermochemical kinetics, noncovalent interactions, excited states, and transition elements: two new functionals and systematic testing of four M06-class functionals and 12 other functionals. Theor. Chem. Acc. 120, 215-241 (2008).

67. F. Weigend; R. Ahlrichs, Balanced basis sets of split valence, triple zeta valence and quadruple zeta valence quality for $\mathrm{H}$ to $\mathrm{Rn}$ : design and assessment of accuracy. Phys. Chem. Chem. Phys. 7, 3297-3305 (2005).

68. A. V. Marenich, C. J. Cramer, D. G. Truhlar, Universal solvation model based on solute electron density and on a continuum model of the solvent defined by the bulk dielectric constant and atomic surface tensions. J. Phys. Chem. B 113, 6378-6396 (2009).

\section{Acknowledgments:}

15 We thank Dr. Xinghua Jin (IAC, SPST, Tianjin University) for conducting and evaluating the NMR analysis, Dr. Jun Xu (IAC, SPST, Tianjin University) for the structure determination of the ligand L3, Ms. Yan Gao for HRMS for all novel compounds. We thank Prof. Jay Siegel (Tianjin University) for fruitful discussions.

\section{Funding:}

National Natural Science Foundation Grant No. 21871207, 22073067 and 21672159

J\&K Scientific Ltd.

\section{Author contributions:}

Conceptualization: JH, YD

Methodology: JH, FZ, LL, CC, YL, GL, SH, MZ, YY, YZ

Investigation: JH, FZ, LL, CC, YL, GL, SH, MZ, YY, YZ

Theoretical calculation: YD, BL

Funding acquisition: JH, YD

Project administration: JH, YD

Supervision: JH, YD

Writing - original draft: $\mathrm{JH}$

Writing - review \& editing: JH, YY, CC, MZ, YD

Competing interests: Authors declare that they have no competing interests.

Data and materials availability: All data are available in the main text or the

\section{Supplementary Materials}

Materials and Methods 
Supplementary Text

Figs. S1 to S9

Tables S1 to S2

References (59-68)

5 